\title{
A Case Study of the Augmentation and Evaluation of Training Data for Deep Learning
}

\author{
JUNHUA DING, University of North Texas, USA \\ XINCHUAN LI and XIAOJUN KANG, China University of Geosciences (Wuhan), China \\ VENKAT N. GUDIVADA, East Carolina University, USA
}

\begin{abstract}
Deep learning has been widely used for extracting values from big data. As many other machine learning algorithms, deep learning requires significant training data. Experiments have shown both the volume and the quality of training data can significantly impact the effectiveness of the value extraction. In some cases, the volume of training data is not sufficiently large for effectively training a deep learning model. In other cases, the quality of training data is not high enough to achieve the optimal performance. Many approaches have been proposed for augmenting training data to mitigate the deficiency. However, whether the augmented data are "fit for purpose" of deep learning is still a question. A framework for comprehensively evaluating the effectiveness of the augmented data for deep learning is still not available. In this article, we first discuss a data augmentation approach for deep learning. The approach includes two components: the first one is to remove noisy data in a dataset using a machine learning based classification to improve its quality, and the second one is to increase the volume of the dataset for effectively training a deep learning model. To evaluate the quality of the augmented data in fidelity, variety, and veracity, a data quality evaluation framework is proposed. We demonstrated the effectiveness of the data augmentation approach and the data quality evaluation framework through studying an automated classification of biology cell images using deep learning. The experimental results clearly demonstrated the impact of the volume and quality of training data to the performance of deep learning and the importance of the data quality evaluation. The data augmentation approach and the data quality evaluation framework can be straightforwardly adapted for deep learning study in other domains.
\end{abstract}

\section{CCS Concepts: • Computing methodologies $\rightarrow$ Machine learning; Cross-validation;}

Additional Key Words and Phrases: Data quality, machine learning, support vector machine, convolutional neural network, deep learning, diffraction image

\section{ACM Reference format:}

Junhua Ding, Xinchuan Li, Xiaojun Kang, and Venkat N. Gudivada. 2019. A Case Study of the Augmentation and Evaluation of Training Data for Deep Learning. 7. Data and Information Quality 11, 4, Article 20 (August 2019), 22 pages.

https://doi.org/10.1145/3317573

\footnotetext{
The authors would like to thank Dr. Xin-Hua Hu, Jiabing Wang, Min Zhang, and Pruthvish Patel at East Carolina University for assistance with the experiments. This research is supported in part by grants \#1560037, \#1730568, and \#1852249 from the National Science Foundation.

Authors' addresses: J. Ding, University of North Texas, Denton, TX, 76203; email: junhua.ding@unt.edu; X. Li and X. Kang, China University of Geosciences (Wuhan), Wuhan, China; emails: lihanyu2006@foxmail.com, 7452822@qq.com; V. N. Gudivada, East Carolina University, Greenville, NC; email: gudivadav15@ecu.edu.

Permission to make digital or hard copies of all or part of this work for personal or classroom use is granted without fee provided that copies are not made or distributed for profit or commercial advantage and that copies bear this notice and the full citation on the first page. Copyrights for components of this work owned by others than ACM must be honored. Abstracting with credit is permitted. To copy otherwise, or republish, to post on servers or to redistribute to lists, requires prior specific permission and/or a fee. Request permissions from permissions@acm.org.

(C) 2019 Association for Computing Machinery.

1936-1955/2019/08-ART20 \$15.00

https://doi.org/10.1145/3317573
} 


\section{INTRODUCTION}

Deep learning (Bengio 2009) has been widely adopted for analyzing big data. A challenging factor for using deep learning is lack of sufficient volume of high quality training data. Many approaches such as crowdsourcing web data (Krause et al. 2015) or transferring data from other domains (Raina et al. 2007) have been proposed for augmenting training data. However, the augmented data probably include noisy data, such as invalid datum and label noise. Although some carefully designed deep learning models are robust to massive label noises (Rolnick et al. 2017), the computing rule of "garbage in, garbage out" is still applied to deep learning. Recently, Rajpurkar et al. introduced a deep learning system called ChexNet for diagnosing pneumonia diseases based on chest X-ray images. They claimed "We find that CheXNet exceeds average radiologist performance on pneumonia detection on both sensitivity and specificity" (Rajpurkar et al. 2017). However, Oakden-Raynera, a radiologist student and machine learning researcher, questioned the dataset used by ChexNet. He said: "I believe the ChestXray14 dataset, as it exists now, is not fit for training medical AI systems to do diagnostic work...., (1) how accurate are the labels, (2) what do the labels actually mean, medically, and (3) how useful are the labels for image analysis"(Oakden-Rayner 2017). Many experiments have shown poor quality data could significantly impact the quality of deep learning (Giannoulatou et al. 2014; Zhang et al. 2013), and some experiments have also shown more training data and better quality of training data could improve the quality of deep learning (Sun et al. 2017). In addition, the overlapped items of data among a training dataset and its corresponding testing dataset could cause overclaimed and misleading results. For example, the significant improvement of the machine learning performance declared in the paper Limsopatham and Collier (2016) published in ACL 2016 probably would not exist if the overlapped datum items were removed from the testing dataset (Lee et al. 2017). Therefore, carefully evaluating a dataset on its effectiveness for deep learning is necessary (Saez et al. 2016).

Many data augmentation approaches have been proposed for producing new data. For example, researchers used a simulation program to simulate the real computing environment for producing training data, such as producing unmanned aerial vehicle data (Mehta et al. 2014) and robot vision data (D’Innocente et al. 2017). Synthetic training data as augmentation data have been used for effectively training deep learning models (Barbosa et al. 2017). Manipulating the existing samples is another simple approach for producing additional samples. For example, new images can be produced through pooling or rotating existing images (Ding et al. 2017a). Simply speaking, transfer learning builds a relation between a limited number of samples and a large volume of related datasets and then applies the relation to the large dataset to produce new samples (Raina et al. 2007). Many generative algorithms (Goodfellow et al. 2014) are also able to produce new training samples. However, generative models and transfer learning are fairly complex for producing large amount of new samples. They may also need a large amount of high quality related training data first before they can be used for producing new samples.

The training data produced using different approaches may contain noisy data such as label noises and duplicates. Machine learning algorithms have been used for detecting duplicates in data that originated from multiple sources (Wu and Song 2015). Data filtering is an approach for improving data quality through removing the noise. Data publishers and subscribers can filter noisy data using domain models and rules (Gudivada et al. 2015). Due to massive scale of big data, automated filtering of noisy data is essential. However, investigations in this direction are just beginning to appear.

In this article, we introduce an automated approach for separating noisy data from a dataset to improve its quality. Our approach includes a machine learning classifier to iteratively separate noisy data from normal data. To address the issue of insufficient volume of training data for 
effectively training the classifier, we experimented different approaches for generating additional training samples from existing data. To ensure the quality of the produced training data, we developed an evaluation approach for evaluating the data quality in veracity, variety, and fidelity. The veracity of the dataset is checked through cross validation of classification accuracy with different classification algorithms, the fidelity of the dataset is checked on the validity of individual samples, and the variety of the dataset is checked through comparing the distribution of the dataset in important parameters. We demonstrate the effectiveness of the proposed approach through studying an automated classification of biology cells based on their diffraction images. The diffraction images of biological cells are taken using a polarization diffraction image flow cytometer (p-DIFC), which is used for quantifying and profiling 3D morphology of single cells (Jacobs et al. 2009).

Research results on systematic evaluation of data quality have been published during the past two decades. Both general purpose and domain-specific techniques and tools have been developed for data quality evaluation and assurance. Wang and Strong (1996) defined a hierarchical framework for defining data quality attributes that has been widely adopted for evaluating data quality. The focus of our work is on the evaluation of the "contextual data quality." which considers the data quality within the context of the task (Wang and Strong 1996). In particular, we only consider the data quality regarding "fit for purpose" for deep learning. We propose a group of quality criteria for evaluating datasets and strategies for the evaluation. The work on data quality built the foundation for evaluation of the quality of big data, which are the typical data type deep learning works on.

The contributions of this research are summarized as follows: First, we propose an approach for improving the data quality through separating noisy data using a deep learning based classification method. The approach can significantly reduce the label noise in datasets. Second, we proposed different data augmentation approaches for producing high volume of training data. The approaches are extremely simpler compared to existing approaches, but the produced data are as authentic as the original data. The most important contribution of this research is due to the systematic approach for evaluating the data quality for deep learning. The evaluation approach can be used for evaluating the data quality for deep learning in any other application.

The remainder of this article is organized as follows: Section 2 provides the domain background in cell imaging and discusses the method and process for automated classification of diffraction images. Section 3 investigates different approaches for data augmentation and experimental results. Section 4 describes an approach for systematically evaluating the quality of augmented data. Related work is discussed in Section 5, and Section 6 concludes the article.

\section{AUTOMATED CLASSIFICATION OF DIFFRACTION IMAGES}

We first discuss the background of morphology-based cell imaging. Next, we discuss morphologybased cell classification using Support Vector Machine (SVM) (Hearst et al. 1998) and deep learning.

\subsection{Data Augmentation and Evaluation of Cell Diffraction Images}

Cells are basic elements of life and possess highly varied and convoluted 3D structures by intracellular organelles to sustain their phenotypic variations and functions. Cell classification is central to many branches of biology and life science research. Label-free and morphology based cell classification such as p-DIFC at the cell level attracts intense research efforts for their direct relations to cellular functions. p-DIFC is used to rapidly acquire cross-polarized Diffraction Image (p-DI) pairs from single cells (Jacobs et al. 2009). It adopts Stokes vectors and Mueller matrices to account for the polarization change in scattered light as a result of intracellular distribution of refractive index and its 3D morphology. The p-DI images thus capture the 3D morphology of the illuminated cells, which is the foundation of the cell classification based on their diffraction images. 
Since an imaging sample of cells may include non-cell particles such as ghost cell body, aggregated spherical particles, and cell debris and small particles, p-DIFC imaging produces images from the non-cell particles in addition to images of viable cells. We refer to the viable cells of intact structures as cells, cell debris and small particles collectively as debris, and ghost cell body and aggregated spherical particles as strip due to the strip texture of its diffraction image. If the images of noncell particles are not removed from the acquired images of the sample, the images will be treated as viable cells. The images of non-cell particles are labeled as cells become label noises in the dataset. If the dataset will be used for training a machine learning model for the cell classification, it is necessary to separate the non-cell diffraction images (i.e., strips and debris) from viable cell diffraction images in the dataset to achieve an optimal classification accuracy. Performing this task manually is not feasible from a practical standpoint. To address this issue, we developed a deep learning approach for classifying the diffraction images into three categories: cells, strips, and debris. The classifier was developed on deep learning architecture AlexNet (Krizhevsky et al. 2012) and TensorFlow framework. The classifier was trained with three classes (i.e., cells, strips, and debris) of labeled diffraction images. Training a deep learning classifier requires a large amount of labeled images for each class. We labeled around 2,000 cell diffraction images for each class, but our experiments showed 10,000 labeled images per class were needed to well train the deep learning classifier (Zhang 2015; Wang 2016). Consulting with domain experts in diffraction images, we developed a data augmentation approach for producing a sufficient amount of diffraction images.

However, whether the augmented data are effective for training a deep learning model is unknown. Therefore, the augmented data need to be systematically evaluated. The evaluation of the augmented data is completed through checking the fidelity, the variety, and veracity of the dataset. We define the fidelity in this context as how close an augmented data sample is to the real one, the variety as how close the distribution of the augmented dataset is to an expected distribution, and the veracity as the improvement of the machine learning performance achieved with the augmented dataset. We proposed a data quality evaluation framework for checking the data fidelity, variety, and veracity in a quantitative way and demonstrated its applicability and effectiveness through an experimental study of the classification of cell diffraction images.

\subsection{Morphology Based Automated Cell Classification}

We first built an SVM classifier based on the textual features of the diffraction images for iteratively labeling unlabeled diffraction images. The SVM classification also serves as a baseline for evaluating the performance of the deep learning classification. Building an SVM classifier and building a deep learning classifier for the classification of diffraction images share the same process. First, identify the labeled datasets for training, validation, and testing. Then iteratively build a classification model. For SVM, it is necessary to first craft a group of features from images for the classification and then experiment with different kernel functions. For deep learning, it is necessary to build a neural network model for learning the features and conducting the classification. Finally, the model is trained and validated. The two classifiers share the same datasets of raw images, but the deep learning classifier is trained and validated with augmented data from the raw images.

\subsection{Datasets}

The three classes of cell particles have different morphology structures, which can be precisely captured in p-DIFC diffraction images with different textural patterns. Based on the textual pattern, a biologist can separate the three different classes of images visually. Figure 1 shows three sample diffraction images and their corresponding particles. The textual pattern of the diffraction image of a viable cell of intact structure contains many bright normal speckles. However, a ghost 


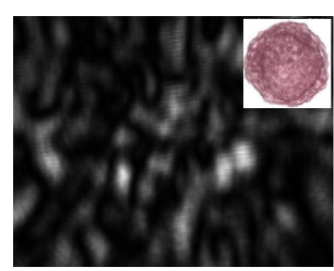

(a)

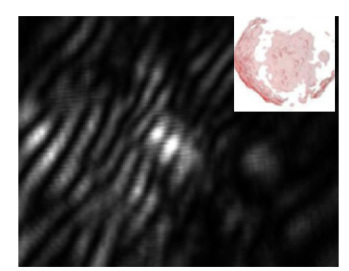

(b)

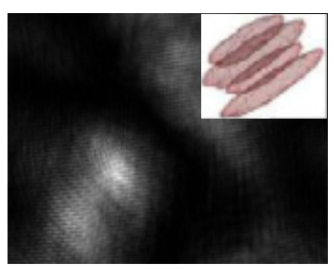

(c)

Fig. 1. A p-DIFC acquired diffraction image of (a) a viable cell of intact structure, (b) a ghost cell body or aggregated spherical particles, and (c) a cell debris or small particle. The top right corner shows the corresponding particle of each image.

cell body or aggregated spherical particle includes bright strips. Last, a cell debris or small non-cell particle shows many large diffuse speckles. The difference in textual patterns of the three classes of diffraction images is the base to separate them using machine learning algorithms. However, the real cases of the textual patterns of the diffraction images could be much more complex. For example, some images of viable cells and debris are similar. Although an expert can label each image in high confidence, label noises do exist in a labeled dataset. Therefore, we used an iterative approach for reducing the label noise. We first manually label a small amount of images for each class, and then train the machine learning classifier using the labeled data. The remaining unlabeled images are entered into the classifier for the classification, and the label of each input image is decided by its classification. The images that are classified with high confidence are then added into the training dataset to retrain the classifier. Labeled images are also randomly selected for manually checking to ensure a reasonable classification accuracy. The incorrect labels are manually corrected. The process is repeated, continuously adding manually labeled images, retraining the classifier with updated training data, and manually checking and correcting the labels. The process continues until the classification accuracy is high enough or it cannot be improved. The approach is effective for labeling a large amount of unlabeled data. It is similar to active learning (Rolnick et al. 2017), but it is much simpler and practical.

We collected a total of 7,519 diffraction images from different cell lines for the case study. Each diffraction image was labeled as cells, strips, and debris accordingly and manually inspected to ensure the correctness. The labeled dataset is composed of 2,232 cells, 1,645 strips, and 3,642 debris.

\subsection{SVM-based Image Classification}

An SVM performs binary classification in general (Burges 1998). To implement a multiclass classification, several SVM classifiers are combined by comparing "one against the rest" or "one against one." We have implemented the classifier for diffraction images using the LIBSVM (Chang and Lin 2011), an open-source toolkit for SVM. We experimented different SVM kernel functions including Gaussian kernel, sigmoid, and Radial basis function (RBF), but RBF achieved the best performance. The SVM experiments discussed in this article were all conducted on RBF.

The textual pattern of the diffraction images is defined using a group of Grey Layer Collaborative Matrix (GLCM) features (Haralick 1971). We use a total of 20 GLCM features. Among them, 14 are the original GLCM definitions and 6 are defined for diffraction images specifically. The definition of each feature can be found in Ding's previous work (Thati et al. 2015).

Our experimental study showed 500 labeled images for each class are sufficient for training the SVM classifier. Increasing the volume of the training dataset more than 500 would not improve the classification accuracy (Thati et al. 2015; Wang 2016). Therefore, some SVM experimental study was conducted on 1,000 labeled diffraction images for each class due to easy calculation. The 
Table 1. Confusion Matrix of the

Classification of Diffraction Images

\begin{tabular}{l||ccc}
\hline & Cells & Debris & Strips \\
\hline \hline Cells & $74.50 \%$ & $16.00 \%$ & $9.50 \%$ \\
\hline Debris & $6.50 \%$ & $81.50 \%$ & $12.00 \%$ \\
\hline Strips & $14.00 \%$ & $24.00 \%$ & $62.00 \%$ \\
\hline
\end{tabular}

average classification accuracy of 10 -fold cross validation (10FCV) for cells, debris, and strips is $74.50 \%, 81.50 \%$, and $62.00 \%$, respectively. The simplified confusion matrix is shown in Table 1 (Ding et al. 2017b). Different feature selection strategies were applied for selecting optimized feature sets (Vilkomir et al. 2017), but none of them could significantly improve the classification accuracy. Other approaches such as pre-selecting the data using image processing and clustering techniques (Zhang et al. 2013) could increase the classification accuracy significantly, but the approach is too complex. It is not fully automated and difficult to be scaled up for the classification of the diffraction images in general. The result shown in Table 1 is a reasonable result an SVM classification can get. But recently, we found deep learning can significantly improve the classification accuracy.

\subsection{Deep Learning Based Image Classification}

Diffraction images are relatively simple due to their low resolution and absence of background noise. Therefore, the deep learning classifier for diffraction images is built on the relatively simple AlexNet model (Krizhevsky et al. 2012). AlexNet is a Convolutional Neural Network (CNN) that won first place at the ImageNet large scale visual recognition challenge in 2012 (ILSVRC2012 2012). It includes multiple convolutional layers for learning features from low-level to high-level, several pooling layers for reducing the complexity of the learned features, and two fully connected layers for the classification. The outputs of the output layer are changed to three classes instead of 1,000 classes in the original AlexNet model. AlexNet was originally trained with about 1.2M images. We did not use the pre-trained AlexNet, since diffraction images are greatly different to the pretrained regular images in ImageNet. The deep learning classifier for classifying diffraction images was trained from scratch with labeled diffraction images. Based on the 7,519 raw diffraction images, data augmentation approaches including cropping or pooling each raw image for producing small images were used to produce large amount of data. Each small image is labeled as its source image, which is the raw image that cropping or pooling is applied to. The model is defined using Python under the Tensorflow framework. It was trained with different configurations such as epochs and optimization strategies. NFCV was used for validation of the classification accuracy. Although we also experimented other CNN architectures from simple ones such as Squeezenet (Iandola et al. 2016) to complex ones like Google Inception architectures (Szegedy et al. 2015), none of them performed better than AlexNet did.

\section{GENERATION OF TRAINING DATA}

The size of a p-DIFC raw diffraction image is $640 \times 480$ pixels. It is large enough to be divided into several small images at size $227 \times 227$ pixels for training AlexNet. Each small image contains sufficient information to represent the original image according to $\mathrm{p}$-DIFC principles (Jacobs et al. 2009). If one checks a diffraction image carefully, it is not difficult to find the textual pattern is repeated in the entire image like those shown in Figure 1. In addition, the textural pattern shown in each image does not simply repeat itself in each section but with slightly changes, which is due to the difference of the view of angle from the imaging camera to each portion of the cell under imaging. The property that a small image cropped from a raw image can capture the textual 


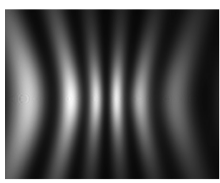

(a)

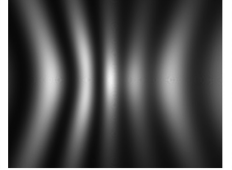

(b)

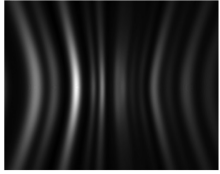

(c)

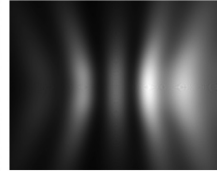

(d)

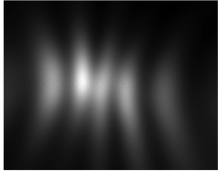

(e)

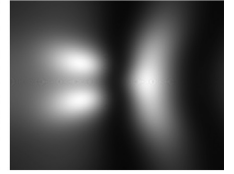

(f)

Fig. 2. aDDA calculated diffraction images of (a) a sphere, (b) an ellipsoid, (c) a bi-sphere, (d) a bi-coated with one sphere in the center, (e) a bi-coated with one sphere shifting from the center, and (f) an RBC.

pattern of the entire raw image at a different view of angle of the imaging camera is the foundation for producing training data through cropping and pooling the raw image. Since a diffraction image may also include large area of black background, which is useless for the classification, a carefully designed approach for avoiding the area is necessary. Although cropping is an effective approach for producing the samples for training the deep learning, it also suffers from some problems. For example, if a textural pattern in an image is too large to be included in a small image, then the cropped images could become a label noise. The case could impact the classification accuracy if they are used for training, but the case is rare. To address the above problem, we also experimented a pooling approach for producing a large amount of small images. Compared to the cropping approach, pooling can preserve the entire textural pattern of a raw image in the small images. However, the resolution of a pooled image is lower than the raw image, since each batch of pixels is represented by one of them.

\subsection{A Preliminary Study of the Training Data Generation Approaches}

To further confirm that a small image is sufficient to capture the morphology information of a cell as a raw diffraction image does, we conducted a simulation based study to demonstrate the soundness of the idea. Program aDDA (add 2016) is an open-source tool for simulating light scattering of particles using discrete dipole approximation (DDA) approach. aDDA can be used to calculate a light scattering of a scatterer. The input to the aDDA is the 3D structure with the refractive index of each component of a scatterer. The aDDA would produce light scattering results in different formats such as Muller matrix, which is used for producing the diffraction image of the scatterer using ray-tracing (Moran 2013). The default size of the calculated diffraction image is set to $640 \times 480$ pixels. Experimental results have shown the textual pattern of an aDDA-calculated diffraction image accurately captures the morphology structure of the scatterer (Moran 2013), which is the theory foundation of p-DIFC. Therefore, we use aDDA-calculated diffraction images to show the relation of the textual pattern between small images and the large one.

We use aDDA-calculated diffraction images to check whether a partial image can represent the whole one. Figure 2 shows six diffraction images calculated from six different scatterers including a homogeneous sphere scatterer (Figure 2(a)) with regular strips that are repeated in the image. If we cut a small window of image such as $300 \times 300$ pixels from the center of an original image, the small image as shown in Figure 3 is sufficient to represent the textual pattern of the original image.

If we shift the window a small distance from the center, we can cut more small images, but each of the images is slightly different. The same property can be observed from other images in Figure 2, which are diffraction images of an ellipsoid scatterer, a bisphere scatterer with two spheres side-by-side, a bi-coated scatterer with one ellipsoid containing a sphere in its center, a bi-coated scatterer with one ellipsoid containing a sphere shifting from its center, and a scatterer for modeling a red blood cell (RBC) in a shape like a peanut. The two spheres in the bisphere scatterer are clearly shown by the repeated strip patterns in the calculated diffraction image, and it is also shown in the corresponding small images in Figure 3. It is easy to see the great similarity 


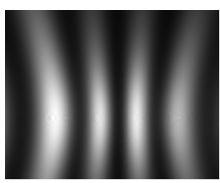

(a)

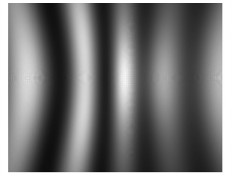

(b)

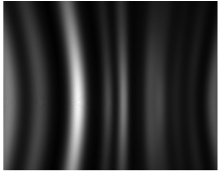

(c)

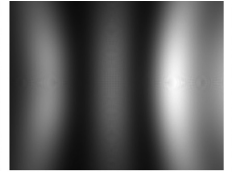

(d)

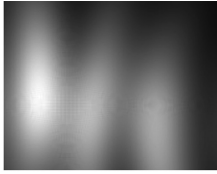

(e)

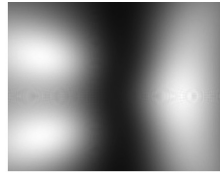

(f)

Fig. 3. Small diffraction images cropped from images in Figure 2. (a) a sphere, (b) an ellipsoid, (c) a bi-sphere, (d) a bi-coated with one sphere in the center, (e) a bi-coated with one sphere shifting from the center, and (f) an RBC.

of the textual pattern between the corresponding images in Figures 2 and 3. From this observation, we believe if we cut small images around the center of the textual pattern in the image, the small images have enough information to represent the original large one.

Now, we calculate the diffraction image of a scatterer at size $300 \times 300$ pixels directly. Nothing needs to be changed for the aDDA calculation, but the only change is the configuration of the ray-tracing simulation, which sets the size of the output image. We found the textural patterns of the large image and the small image produced from the same aDDA simulation result is exact same except the difference of the image resolution. We conclude the small size diffraction image is sufficient to capture the morphology information of a scatterer.

\subsection{Cropping Images}

As noted earlier, AlexNet accepts input images at size $227 \times 227$ pixels. Also, the size of a raw diffraction image is $640 \times 480$ pixels. A small image is about $1 / 5$ of the size of a raw image. Furthermore, since a diffraction image may contain black areas, the center of the textual pattern such as bright speckles or strips may not be in the center of the image. It is important to find the center of the textual pattern area to perform cropping. The center is normally the brightest area in an image. An experimental approach based on sliding window was developed for finding the center of a textural area in an image. Given a $5 \times 5$ pixel window, the cropping program calculates the average intensity of the window. Then it slides the window with a stride at 5 pixels first horizontally and then vertically to cover the entire image, to find a window that has the highest average intensity. The window with the highest average intensity is set as the center for cropping small images. If multiple windows have the same highest average intensity, the one nearer to the center of the raw image is selected. A small image at the preset size is cropped from the raw image around the center first, and then more small images are cropped through sliding the window from the center a certain number of pixels in any direction as soon as the cropped image is within the raw image.

In our study, the $227 \times 227$-pixel window is moved from the center in eight different directions. The angle $\theta$ between two adjacent directions is $45^{\circ}$. The sliding window moves $d$ pixels ranging from 7 to 17 pixels from the last window in a particular direction to crop a new image. The sliding window can be moved several strides in one direction to crop multiple small images in that direction. When a sliding window is moved to a new position, it is necessary to ensure that the entire sliding window is still contained in the original image boundary. If not, the window is discarded and no further sliding in that direction takes place. Finally, 105,291 small diffraction images of cells, 127,733 of debris, and 92,767 of strips are cropped and selected for training the deep learning classifier.

\subsection{Pooling Images}

The cropping technique does not work well for the case where the whole image is critical for the classification. In such a case, local features that are extracted from a local area are not enough to 


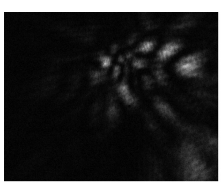

(a)

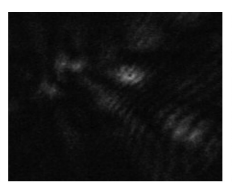

(b)

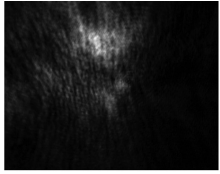

(c)

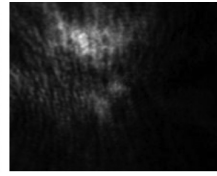

(d)

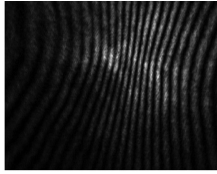

(e)

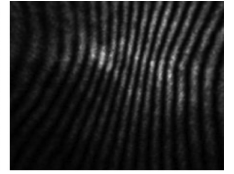

(f)

Fig. 4. Diffraction images and their pooling images. (a) and (b) are a cell, (c) and (d) are debris, (e) and (f) are a strip.

represent the global features extracted from the whole image. We experimented a pooling technique for producing a large volume of training data. A raw diffraction image is downsampled into a small image using pooling. Multiple small images can be produced from a raw image with different pooling configurations. Also, small images can be produced with different pooling functions such max pooling and average pooling (Dee 2017a).

To produce multiple small diffraction images from a raw image, we apply different pooling window sizes and different sliding strides to the same image. Since the size of the small image is $227 \times 227$ pixels and the size of a raw image is $640 \times 480$ pixels, we resize the raw image into a square one. We cut three different squares from an image, which are $455 \times 455,456 \times 456$, and $457 \times 457$ pixels, respectively. Next, $3 \times 3,4 \times 4$, and $5 \times 5$ pixels pooling windows are applied to the three squares. The stride distance is set to 2 pixels. The size of the output image from the pooling is $s * s$ pixels, $s=(x-m) / c+1$, where $x \times x$ pixels are the size of the input image of the pooling, $m \times m$ pixels are the size of the pooling window, and $c$ is the stride distance. For example, if the input image is $455 \times 455$, the pooling window is $3 \times 3$ pixels, and stride distance is 2 , the size of the output image will be $227 \times 227$ pixels. With different pooling configurations, each small image pooled from the same raw image would contain unique texture. Figure 4 shows a comparison of the raw images and their pooling images (the images were resized with different ratios for better formatting). It clearly shows the textual pattern of a raw image is well preserved in the pooling images.

We experimented three pooling functions, including average-pooling, max-pooling, and minpooling. However, each dataset includes only the small images that were pooled from raw images using the same pooling function.

\subsection{Experimental Results}

In this section, we discuss the experimental results of the deep learning classification with different datasets. We also discuss the experimental results of the SVM classification with the new datasets. $8 \mathrm{FCV}$ and confusion matrix were used to validate the classification results. Many experiments were conducted for optimizing the classifiers and validating the classification results, but we only describe the important results.

3.4.1 Experimental Results with Cropped Images. 8FCV shows the average classification accuracy of viable cells at $99.36 \%$, debris at $97.74 \%$, and strips at $99.81 \%$. Figure 5 shows the confusion matrices of four sample groups of classification results from the 8FCV. From 8FCV results, we note that the classifier built on AlexNet is highly accurate for classifying the three classes of diffraction images. Also, the dataset produced from the raw images is sufficient for training the classifier.

3.4.2 Experimental Results with Pooled Images. The 8FCV result of the classification based on the dataset generated using average-pooling shows that the average classification accuracy for debris and strips is a little bit higher than the dataset built by cropping. However, the average classification accuracy for cells is much lower at $85.7 \%$ vs. $94.22 \%$. As shown in Table 2, nearly $10 \%$ of cells were incorrectly classified as debris, and $4.5 \%$ were incorrectly classified as strips. 


\begin{tabular}{|c|c|c|c|}
\hline & cell & debris & strip \\
\hline cell & 99.02 & 0.62 & 0.37 \\
\hline debris & 1.89 & 97.19 & 0.92 \\
\hline strip & 0.06 & 0.18 & 99.76 \\
\hline cell & 99.63 & 0.37 & 0 \\
\hline debris & 1.99 & 97.19 & 0.82 \\
\hline strip & 0.47 & 0.24 & 99.29 \\
\hline cell & 99.26 & 0.62 & 0.12 \\
\hline debris & 0.92 & 98.57 & 0.51 \\
\hline strip & 0.06 & 0.12 & 99.82 \\
\hline cell & 99.38 & 0.55 & 0.06 \\
\hline debris & 0.92 & 98.82 & 0.26 \\
\hline strip & 0 & 0.18 & 99.82 \\
\hline
\end{tabular}

Fig. 5. Four groups of confusion matrices of a classification experiment.

Table 2. A Confusion Matrix of a Deep Learning Classification with an Average-pooling Dataset

\begin{tabular}{l||ccc}
\hline & Cells & Debris & Strips \\
\hline \hline Cells & 0.857 & 0.098 & 0.045 \\
\hline Debris & 0.006 & 0.987 & 0.006 \\
\hline Strips & 0.005 & 0.052 & 0.943 \\
\hline
\end{tabular}

Table 3. A Confusion Matrix of a Deep Learning Classification with a Min-pooling Dataset

\begin{tabular}{l||ccc}
\hline & Cells & Debris & Strips \\
\hline \hline Cells & 0.935 & 0.036 & 0.030 \\
\hline Debris & 0.024 & 0.961 & 0.015 \\
\hline Strips & 0.023 & 0.044 & 0.933 \\
\hline
\end{tabular}

We note that the difference between the textual patterns in a diffraction image of a viable cell and debris is the size of speckles-a debris image normally has larger diffuse speckles. The averagepooling would decrease the difference between a viable cell and debris, which could be a reason that more viable cells were classified as debris. The $8 \mathrm{FCV}$ results and confusion matrices further confirm that the deep learning classification is more effective than the SVM classification. However, the dataset created using average-pooling could be improved. Therefore, we also experimented with max-pooling and min-pooling functions for generating training data.

The $8 \mathrm{FCV}$ result of the classification based on the dataset created using max-pooling is almost identical to the result shown in Table 2. Average classification accuracy of cells is $87.9 \%$, of debris is $98.5 \%$, and of strips is $94.6 \%$. However, the $8 \mathrm{FCV}$ result of the classification based on the dataset created using min-pooling is much better. The average classification accuracy of cells is improved to $93.5 \%$, of debris to $96.1 \%$, and of strips to $93.3 \%$. The confusion matrix is shown in Table 3 . Figure 6 shows a comparison of the average-pooling images and their corresponding min-pooling images. We found that the textual patterns in min-pooling images are much clearer, which might be an explanation of the improvement of the classification accuracy. 


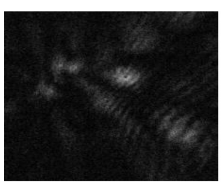

(a)

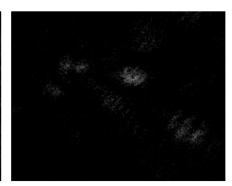

(b)

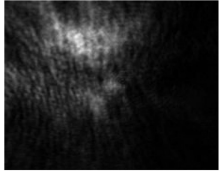

(c)

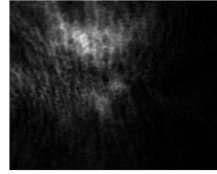

(d)

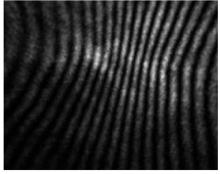

(e)

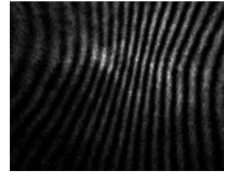

(f)

Fig. 6. Diffraction images produced with different pooling functions. (a) an average-pooling image of a cell, and (b) the corresponding min-pooling image of (a); (c) an average-pooling image of the debris, and (d) the corresponding min-pooling image of (c); (e) an average-pooling image of a strip, and (f) the corresponding min-pooling image of (e).

Table 4. A Confusion Matrix of an

SVM Classification with a

Min-pooling Dataset

\begin{tabular}{l||ccc}
\hline & Cells & Debris & Strips \\
\hline \hline Cells & 0.995 & 0.005 & 0.000 \\
\hline Debris & 0.081 & 0.915 & 0.004 \\
\hline Strips & 0.029 & 0.121 & 0.850 \\
\hline
\end{tabular}

3.4.3 Experimental Results of SVM Classification with Small Images. The datasets used for the deep learning classification are different from those used for the previous SVM classification, since the SVM classification was conducted on the raw images and the deep learning classification was done on the updated datasets consisting of small images. It is interesting to know whether the SVM classification can result in a different classification accuracy if it is trained on the datasets as the deep learning classification did. We ran the same min-pooling program that was used for producing the datasets for the deep learning and produced the same number of small images for each class as the datasets for the deep learning classification. Only 10,000 labeled images of each class were selected for the dataset, since they are more than enough for training the SVM classifier. The SVM classifier was trained with the 30,000 labeled GLCM feature vectors and $8 \mathrm{FCV}$ was conducted. The confusion matrix in Table 4 shows the classification accuracy of the $8 \mathrm{FCV}$ on the 30,000 diffraction images.

The accuracy of the SVM classifier with the min-pooling dataset was improved considerably compared to the results with raw images. The accuracy is comparable to the deep learning classifier as shown in Table 3. Can we conclude that the dataset produced via min-pooling would train the classifier much better than the raw image dataset? We suspected the hypothesis, since the images of the min-pooling dataset were pooled from the raw images. The pooled images do not add new information to the dataset. The change of the size of the dataset from 3,000 images to 30,000 should not contribute anything to the accuracy improvement based on our previous experimental results. Then what went wrong and how can we identify the cause?

3.4.4 What Went Wrong? Although the SVM classification and the deep learning classification used the same raw images, the datasets produced from pooling the raw images for the two experiments were also produced using the same Python program, but the experiments were conducted separately by different researchers in different servers. Therefore, we decided to conduct a deep learning classification using the exact same datasets as the one used for the SVM classification in the same server by the same researcher. The average accuracy of 8FCV is shown in Table 5.

The accuracy of $8 \mathrm{FCV}$ of the deep learning classification on the dataset used for the SVM classification is much higher than the accuracy of the deep learning classification we did before. From the 
Table 5. A Confusion Matrix of a

Deep Learning Classification with a Min-pooling Dataset

\begin{tabular}{l||ccc}
\hline & Cells & Debris & Strips \\
\hline \hline Cells & 1.000 & 0.000 & 0.000 \\
\hline Debris & 0.001 & 0.999 & 0.000 \\
\hline Strips & 0.000 & 0.000 & 1.000 \\
\hline
\end{tabular}

results shown in Table 5, it is not difficult to suspect that the classifiers suffered from overfitting. It is highly possible that the dataset we used in the latter experiments has some problem.

To investigate the overfitting problem, we acquired new diffraction images using a p-DIFC instrument with carefully prepared blood samples. Then we selected and labeled 100 new acquired diffraction images for each class (i.e., cells, debris, and strips). Each image is converted into a small image using the min-pooling program. The new image dataset was used for testing the accuracy of both the SVM classifier and the deep learning classifier. The classification accuracy of the new test dataset using the SVM classifier that was trained using the min-pooling dataset was $69.1 \%$, $72.5 \%$, and $60.9 \%$ for cells, debris, and strips, respectively. The classification accuracy of the new test dataset using the deep learning classifier that was trained using the latest min-pooling dataset was $90.7 \%, 93.5 \%$, and $89.7 \%$ for cells, debris, and strips, respectively. Both results were consistent to the SVM classification on raw images and the deep learning classification on the previous min-pooling dataset. Based on the experimental results, it is easy to note that the dataset used for previous and the latter experiments are different. But all experiments used the same raw images and the Python program for producing the small images. What went wrong? We checked the pooled images from the same raw image produced in previous experiments and the latter one by comparing the corresponding images pixel-by-pixel. (The raw image and its pooled images are linked by their file names. For example, if the file name of a raw image is P00001.BMP, then its pooled images will be named as P00001-001.BMP, P00001-002.BMP, ...) The pooled images produced by all experiments were exactly the same, but we found that the test images in the previous experiments and the those used in the latter one were different. In the previous experiments, the raw images were first divided into eight groups, and one group was reserved for testing each time. The grouping of the pooled images was decided by the grouping of the raw images. In other words, the small images produced from the testing data group of the raw images will stay in the testing group, and the small images produced from the training data groups of the raw images will stay in the training data groups in the pooled image datasets. In the latter experiments, the pooling was applied to all raw images first, and then the small images were divided into eight groups, and one group was used for the testing each time. Because one raw image can produce multiple small images through pooling, it is highly possible that the small images produced from the same raw image were grouped into both the training data groups and the testing data group. For example, raw image $A$ produced 10 small images, and then 2 of them could be put into a testing data group and 8 of them could be put into the training data groups. In the previous experiments, the small images produced from the same raw image are either put into the training data groups or into the testing data group altogether, but it is impossible that they are split into the training data groups and the testing data groups. Although these small images produced from the same raw image present slightly different textual patterns due to the difference of their imaging angles, they are still significantly similar in textual patterns or essentially identical, because they could be transformed into the same textual pattern. Since the small images were shuffled to ensure even distribution in the dataset, for each image in the testing data group, there are around seven similar images in the training data groups in the 
latter experiments. The $8 \mathrm{FCV}$ cannot solve the problem, since these images from the same raw image are evenly distributed into all groups through shuffling. We concluded that both the deep learning classifier and the SVM classifier remembered these images and caused the overfitting.

3.4.5 What Did We Learn? The experiments we discussed in the above sections have shown that data quality can significantly impact the performance of both traditional machine learning and deep learning. In the special case, the overlapped data between the training and testing datasets significantly "increased" the classification accuracy due to overfitting. If the classifier is not further validated using new data, the problem would not be easily detected. Then, if the trained classifier is used for classifying samples from real applications, many classification errors would be produced. This problem is more pronounced for deep learning, since it usually requires a large amount of training data. For example, AlexNet was trained using around 1.2M images from ImageNet. These images were collected and labeled through crowdsourcing, which probably includes many label noises as discussed by Warden in Warden (2018). It is also possible that some of the testing data already exist in the training data. These items of data could be named differently, or they probably are stored in different formats. If the number of overlapped samples is high, it could cause an overfitting problem or over-claimed performance. During the revision of this article, we found another example that confirmed the impact of the quality of datasets to the performance of machine learning. The experimental result reported in Limsopatham and Collier (2016) showed up to $44 \%$ performance improvement compared to the last best result. However, we found a significant amount of testing items of data also exist in the training dataset. For example, in TwARD-L dataset, around $60 \%$ of the testing items of data in each group also exist in the corresponding training data groups. If the overlapped items of data are removed from the testing data, our experimental results showed the proposed approach discussed in Limsopatham and Collier (2016) will not improve any machine learning performance compared to the baseline used in the paper. The same conclusion was also confirmed in Lee et al. (2017) by another research group. In the following section, we will introduce a framework for evaluating the quality of datasets to address the above issues.

\section{AN APPROACH FOR EVALUATING THE QUALITY OF AUGMENTED DATA}

There are many approaches for evaluating data quality (Batini et al. 2009; Daniel et al. 2018), but the work directly related to deep learning is rare. In this section, we discuss how to systematically evaluate the quality of augmented datasets, which are produced from raw diffraction images using cropping or pooling techniques for deep learning. We evaluate the quality of a dataset in veracity, fidelity, and variety. We define the three terms considering "fit for purpose" for machine learning. The definitions are slightly different from the definitions in other data quality publications such as the widely cited paper authored by Wang and Strong (1996). The three terms used in the paper are the best for defining the data quality we mean. "Veracity" means that the augmented dataset contains all information in the raw/original dataset and it can represent the original dataset to train a machine learning model. "Fidelity" refers to the fact that a generated datum cannot be distinguished from a real one based on meaningful properties. For example, one cannot differentiate a cropped diffraction image and a raw diffraction image based on their GLCM values or textual patterns. "Variety" means an augmented dataset should show the same distribution as the original dataset in meaningful properties, although the two distributions probably have different values in the meaningful properties. For the diffraction image case-study, first we check the textual pattern of the small images to ensure the small image can capture enough morphology information as its original one. Then, we compare the distributions of meaningful feature values of the augmented dataset and its corresponding original dataset. Finally, we investigate whether the small diffraction images can be used for classifying the diffraction images to achieve the similar accuracy as 
the original images. Based on the case study results, we propose a framework for evaluating the quality of datasets for deep learning. The framework is summarized as follows:

(1) Check the fidelity of newly produced individual samples. This task is to verify that each newly produced sample is as authentic as a real one. It also needs to check the uniqueness of each produced sample. It can be completed by visually checking the individual samples for any anomalies, comparing the parameter values of newly produced samples and real samples, or checking the newly produced samples using an anomaly detection tool. For example, one can check the textual pattern of newly produced diffraction images to identify any samples with invalid textual patterns. One also can check whether the value of each GLCM parameter of an image is within the valid range. Some statistic tools such as IBM SPSS and GNU PSPP can be used to check the uniqueness and the anomalies of the newly produced samples (Batini et al. 2009).

(2) Check the variety of a newly produced dataset. This task is to check if a newly produced dataset has a compatible distribution as the original dataset in selected parameters. For example, both datasets may follow the same uniform distribution in selected parameters, although the two datasets may have different values in the parameters. In the diffraction image case, the image intensity of the new augmented dataset and raw image dataset should follow a normal distribution, but they have different variances and slightly different mean values. The task can be completed through generating and comparing the distributions of an augmented dataset and the corresponding original dataset. Based on the domain knowledge, one can define the expected distribution of a dataset in a selected parameter. In most cases, uniform distribution and normal distribution should first be checked. The other approach is to plot a $2 \mathrm{D}$ or $3 \mathrm{D}$ plot of a dataset, and then one can visually examine the separation of the samples by the selected parameters. The plots of two datasets then can be used to check whether the samples are separated in a similar way by the selected parameters.

(3) Check the veracity of a newly produced dataset. This task is to check if a newly produced dataset can be used for training a machine learning model as the original dataset. Of course, the machine learning model used for the data quality evaluation normally is a relatively simple one so it can be well trained with both the newly produced dataset and the limited amount of original data. The performance of the machine learning model trained with the datasets might not be optimal, but it is sufficient for checking whether the machine learning model trained with the new dataset could achieve the same performance as the original one. For example, we trained an SVM classification using the small image dataset and then trained the model from scratch with the raw image dataset. Both classifications had similar classification accuracy for each class, which showed the newly produced dataset was as effective as the raw image dataset for training the machine learning model.

\subsection{Checking the Fidelity of Newly Produced Individual Samples}

Using the diffraction images as an example, we explain how to check the fidelity of the small images produced from cropping and pooling the raw diffraction images. First, we arbitrarily select a group of small images from each class and visually inspect each to see if it presents the expected pattern: the normal speckles of cell images, the strip pattern of strip images, and large diffuse speckles of debris images. If no anomaly is found, then we check the GLCM feature values for each image, since the textual pattern is essential to the classification of diffraction images. Then, we check whether the GLCM values of each small image are within the corresponding GLCM value 
Table 6. A Sample Comparison of GLCM Feature Values Among Diffraction Images

\begin{tabular}{l||cccccc}
\hline & ASM & CON & COR & VAR & IDM & SAV \\
\hline \hline Img-1 & 0.069028397 & 0.300829789 & 0.978817148 & 0.164436833 & 0.597584362 & 0.231334924 \\
\hline Img-2 & 0.656232866 & 0.224157652 & 0.980417054 & 0.130393754 & 0.864684597 & 0.109085359 \\
\hline Img-3 & 0.967753732 & 0.017326016 & 0.688163634 & 0.000792628 & 0.989551474 & 0.026260393 \\
\hline Img-4 & 0.026913115 & 0.159871456 & 0.99144913 & 0.166720538 & 0.621992081 & 0.292738468 \\
\hline Img-5 & 0.587408623 & 0.235948351 & 0.951577511 & 0.063657455 & 0.836049419 & 0.093207132 \\
\hline Img-0 & 0.329891354 & 0.001331282 & 0.997723423 & 0.117280715 & 0.84966343 & 0.282349741 \\
\hline
\end{tabular}

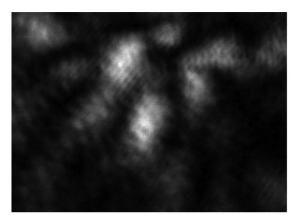

(a)

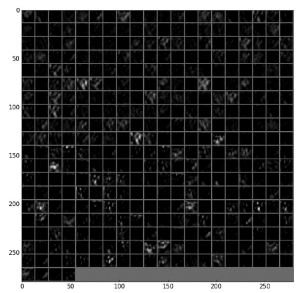

(d)

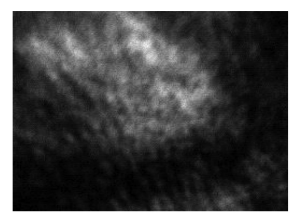

(b)

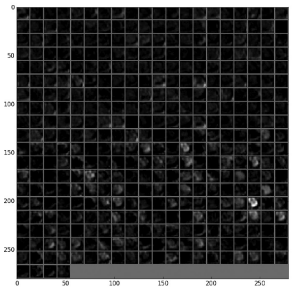

(e)

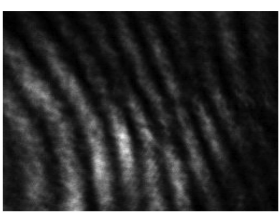

(c)

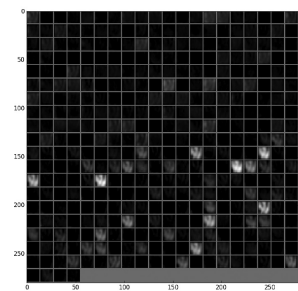

(f)

Fig. 7. Sample input images for AlexNet and their corresponding feature maps output from the last convolutional layer. (a) and (d) are a cell, (b) and (e) are debris, and (c) and (f) are strips.

ranges calculated from its original image dataset. If any GLCM feature value of a small image is out of the range significantly, it should be manually checked. Finally, we check if there are any redundant samples in the dataset. We compare the GLCM feature vectors of all small images. If any two images have the same GLCM values for all features, then the two images are considered as the same. The feature analysis process can be easily implemented using a statistical software tool such as IBM SPSS or GNU PSPP. Table 6 shows a sample comparison of pooled small images and its original image in six GLCM features. Img - 1 to Img -5 are pooled images from raw image Img - 0. It shows each image has different GLCM values.

To further check the fidelity of individual images, a good way is to check the learned feature of each small image in the deep learning. For example, given a small image for each class, one can check how the image features are learned from low-level features to the high-level features in AlexNet. Figure 7 shows three small images in three different classes and their corresponding feature maps output from the last convolutional layer of AlexNet, which is supposed to extract the high-level features before the pooling layer and the two fully connected layers in the network. From the figure, it is easy to find the features learned from the small images are sufficient to separate the three classes of images. The fidelity of the three small diffraction images compared to the original images is high. We recently also implemented a simple strategy to automate the process. The feature map of an image is vectorized as a length 4,096 vector, and then a hash function 

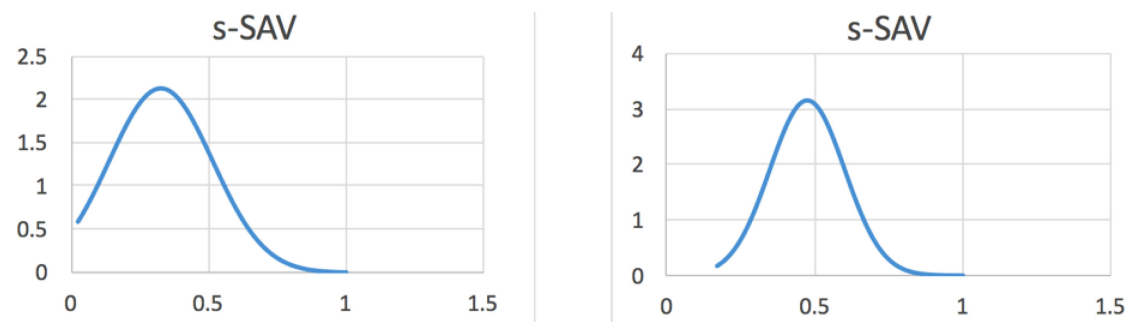

Fig. 8. A comparison of the distribution of a dataset of original images on a GLCM feature (i.e., the right diagram) and a dataset of small images pooled from the original images (i.e., the left diagram).

is applied to the vector to compute a hash value. A small image and its original raw image are entered into the AlexNet to calculate the hash values for both images. The two images should have similar hash values. The strategy can be used for checking any type of data as soon as they can be processed by a CNN. The method provides an effective and efficient way to check the fidelity of augmented data.

\subsection{Checking the Variety of a Newly Produced Dataset}

An effective approach for checking the variety of a dataset is to create a distribution such as normal distribution of a dataset on a selected parameter and then check whether the samples are well distributed as expected. Many software tools such as IBM SPSS or GNU PSPP can be used for computing and visualizing the distribution of a dataset. Visualizations such as distribution diagrams and $\mathrm{Q}-\mathrm{Q}$ plots are effective tools for understanding the distribution. In many cases, the data will not be perfectly distributed as expected, since real-world data could include "rare" samples or outliers. As soon as a distribution can be explained by the domain knowledge, the dataset is acceptable regarding the selected parameters. The selection of distribution parameters could be challenging, since deep learning does not need hand-crafted high-level features for the training. For example, the input to the deep learning model for the classification of diffraction images is the image itself. There is no way to create a distribution based on images directly. In our case, we use GLCM parameters for building the distribution. In other cases of images, other feature parameters such as wavelets or contourlets or other signal-processing parameters can be used to extract features. For sequence data such as natural language data, a data sample can be converted into a feature vector such as those representations in Word2Vec or GloVe. These features might not be sufficient for a deep learning based classification, clustering, or regression analysis, but they are good enough for checking the variety of a dataset. Normally, we select the parameters as simple as possible as soon as each sample has different value on the parameter. The same distribution of the original dataset serves as a reference distribution for the newly produced dataset. However, the two distributions are not necessary to be identical, since some parameters are related to the size of the dataset and the resolution of the items of data.

The left diagram in Figure 8 shows a distribution of a group of 3,250 small images on GLCM parameter s-SAV, and the diagram on the right is the corresponding distribution on the same parameter of the original 2,167 diffraction images that the small image pooled from. The two distributions have different mean and deviation values, but the two distributions have the same trend on the parameter values, and the data samples from the small image dataset are fairly well distributed within the value range. We conclude the small image dataset holds the variety property in parameter s-SAV. 

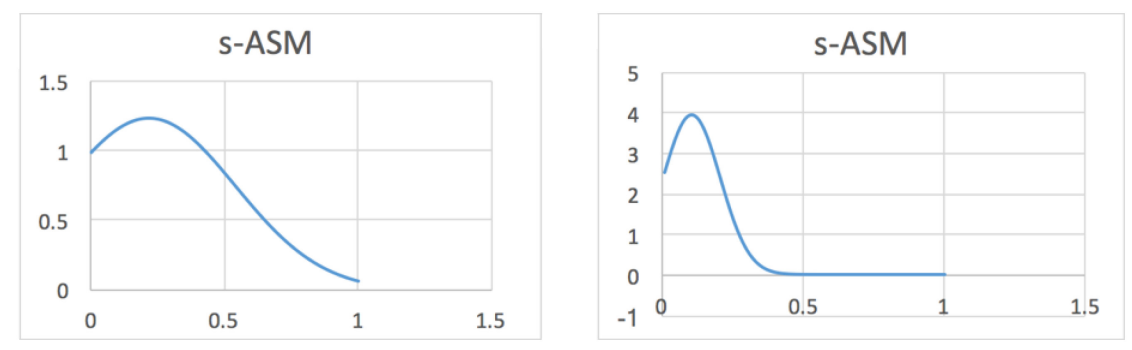

Fig. 9. A comparison of the distribution of a dataset of original images on a GLCM feature (i.e., the right diagram) and a dataset of small images pooled from the original images (i.e., the left diagram).
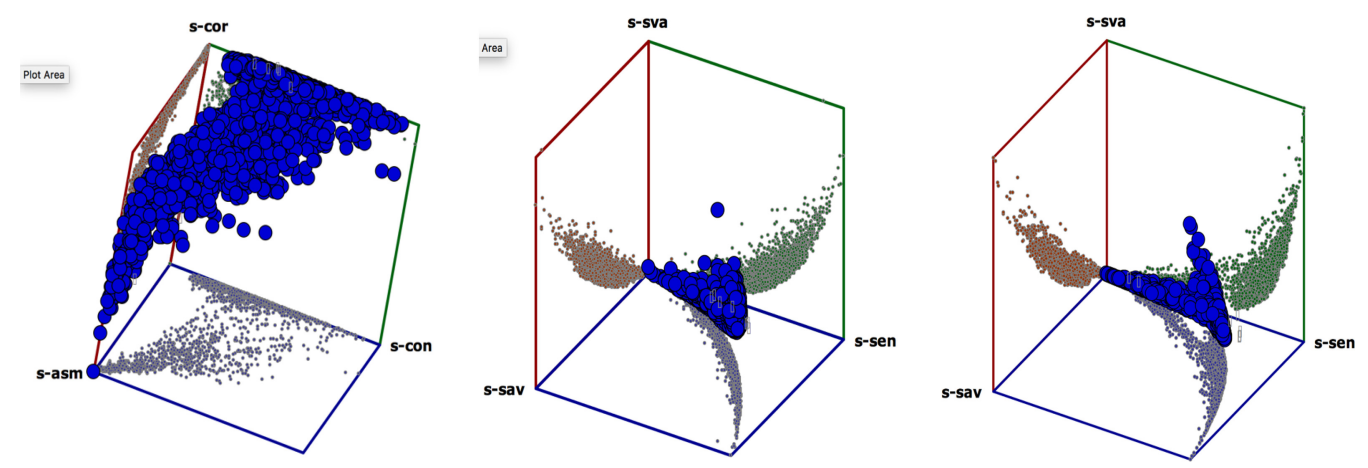

Fig. 10. Plot diffraction images in a 3D space with three GLCM feature parameters. The left diagram shows a group of small images. The right diagram shows a group of original images, and the middle diagram shows a group of small images pooled from the original images. The right and middle diagrams are plotted using the same GLCM feature parameters, but the left one is plotted using different GLCM parameters. The left diagram and the middle diagram show the same group of small images in different GLCM feature parameters.

Figure 9 shows two distributions created from the same datasets used in Figure 8 but with a different GLCM parameter: s-ASM. The left diagram is the distribution of the small image dataset, and the right one is the distribution of the raw image dataset. The two distributions look very different. However, if we check them carefully, it is not difficult to see that the two distributions have the same trend. For example, much more samples are located on the right side of the mean than the left side. The samples in the dataset of the small images are more variable within the value range of parameter s-SAM than the original dataset. In this case, we believe the dataset of the small images has better quality in variety, which is exactly needed for effectively training a deep learning model.

Plotting the samples from a dataset into a $2 \mathrm{D}$ and $3 \mathrm{D}$ plot with selected parameters is also an effective way for checking the variety of a dataset. For example, we plotted the samples of a dataset of small images with GLCM parameters s-ASM, s-CON, and s-COR. The samples are plotted in the $2 \mathrm{D}$ planes and a 3D space as shown in the left diagram in Figure 10. It is not difficult to see the samples are well distributed in the 2D planes and the 3D space.

We also compared the plots of the samples in the dataset of the small images and the samples of the dataset of the original diffraction images to see whether the varieties of the two datasets are consistent. The two plots were plotted with the same GLCM parameters, and both datasets had similar number of samples. Checking the middle diagram and the right one in Figure 10, it 


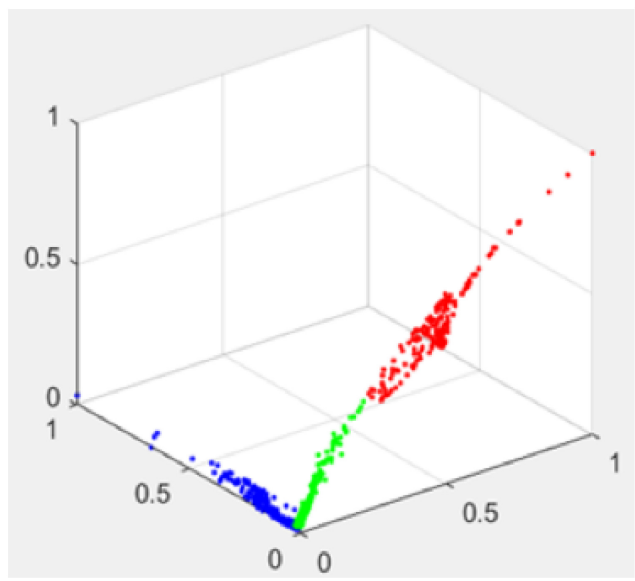

Fig. 11. A GMM clustering of 600 small diffraction images that were pooled from raw images. The three different colors show three different classes of the diffraction images, which are cells, strips, and debris.

is not difficult to conclude that the dataset of the small images enjoys higher variety than the original image dataset through checking the 2D and 3D sample scattering. Through checking the distributions of a newly produced dataset and visually inspecting the sample scattering in plots, one can better understand the variety of a dataset.

\subsection{Checking the Veracity of a Newly Produced Dataset}

The purpose of checking the veracity of a newly produced dataset is to validate whether the newly produced dataset can be used for training the deep learning model. The straightforward way is to train the deep learning model using the new dataset and then test the performance using a testing dataset. However, training a deep learning model is normally complicated. Therefore, a simple method should be developed. For example, one can experiment a clustering method to show whether the new dataset can be clustered as expected, or a logistic regression based on selected features to see whether the samples in the dataset can be grouped in some degree. In many cases, we compare the performance of the clustering or classification conducted on the original dataset and the augmented dataset to see whether the new dataset could achieve the similar performance. If it does, the new dataset should be as effective as the original dataset for training the deep learning.

We conducted a clustering and a classification experiment on a small image dataset. 200 small diffraction images that were pooled from raw diffraction images were selected for each class, and they were mixed together to conduct a clustering analysis. The reason for choosing only 600 images is to minimize the validation cost. The clustering was conducted on the GLCM feature values of the small images. We conducted a clustering based on K-Mean algorithm with $\mathrm{K}=3$ to represent the three classes of images. However, the average accuracy of the clustering was around $45 \%$, which is not good. Then we experimented the clustering using GMM (Gaussian Mixture Models) algorithm (Sridharan 2014) with the same GLCM vectors. The samples were well separated by GMM with average accuracy of $90 \%$. Figure 11 shows a visualization of the GMM clustering result.

The classification of the dataset of small images was conducted using an SVM classification based on GLCM feature values, the SVM classification we discussed in previous sections. We selected 1,000 small images for each class and then conducted an 8FCV. The confusion matrix of the average result of $8 \mathrm{FCV}$ is shown in Table 7 . Comparing the results in Tables 7 and 1, which is 
Table 7. Confusion Matrix of an SVM Classification of the Augmented Dataset

\begin{tabular}{l||ccc}
\hline & Cells & Debris & Strips \\
\hline \hline Cells & $77.94 \%$ & $10.97 \%$ & $11.09 \%$ \\
\hline Debris & $7.33 \%$ & $84.39 \%$ & $8.28 \%$ \\
\hline Strips & $20.25 \%$ & $18.69 \%$ & $61.06 \%$ \\
\hline
\end{tabular}

the SVM classification result of the original dataset, it is easy to find the classification accuracies of the two training datasets are almost identical. We conclude that the augmented dataset accurately represents the original dataset for training machine learning models based on the clustering and classification results. It provides evidence to demonstrate the high veracity of the augmented dataset for deep learning.

\section{RELATED WORK}

Data quality is critical to a machine learning system, because poor data could cause serious problems such as wrong prediction or low classification accuracy. Wang and Strong (1996) developed a hierarchical framework for defining data quality attributes in four categories: intrinsic data quality, contextual data quality, representational data quality, and accessibility data quality. They concluded that "high quality data should be intrinsically good, contextually appropriate to the task, clearly represented, and accessible to the data consumer" (Wang and Strong 1996). The focus of our work is on the contextual data quality of datasets for deep learning. We defined three quality attributes regarding the data fidelity, variety, and veracity that would impact the performance of deep learning. The framework defined by Wang and Strong has been widely adopted for quality evaluation of big data (Cai and Zhu 2015; Gudivada et al. 2015), which is the case for deep learning most of the time. Gao et al. provided an overview of big data quality issues, discussed challenges, and evaluated tools for validation and quality assurance of big data (Gao et al. 2016). They defined big data quality assurance as the study and application of quality assurance techniques and tools to ensure the quality attributes of big data (Gao et al. 2016). Although many general techniques and tools have been developed for quality assurance of big data, domain-specific techniques are typically needed. For example, data quality assurance in the healthcare domain is quite different from those in biomedical sciences or banking and financial services. Furthermore, the data quality requirements for deep learning is different than other applications. We simplified the data quality attributes into the three most important ones for deep learning: fidelity, variety, and veracity of a dataset. Batini et al. gave a comprehensive review of the methodologies for data quality assessment and improvement (Batini et al. 2009), but the application domain is limited to database applications. Crowdsourcing is an effective and widely adopted way for collecting a large amount of data for deep learning (Krause et al. 2015). The results reported in Krause et al. (2015) showed noisy data could be highly effective for training a deep learning model. But we believe if noisy data are removed from a training dataset and it is evaluated for fidelity, variety, and veracity, the performance and confidence of the deep learning could be even higher. Daniel et al. recently conducted a survey on the quality attributes, assessment techniques, and assurance actions of crowdsourcing (Daniel et al. 2018). However, the focus of the article was on the evaluation of crowdsourcing project development processes and products in general. The evaluation approach cannot be directly used for evaluating the quality of a dataset for deep learning. Sun et al. have shown how the size and quality of a dataset would impact the performance of deep learning (Sun et al. 2017; Warden 2018). 
Some preliminary work on the evaluation of the veracity, variety, and fidelity of data were reported, although the definitions of the attributes could be slightly different due to the difference in the application domain. There are many investigations on evaluating the veracity of web sources using hyperlinks, browsing history, and the factual information provided by the source (Dong et al. 2015a). Furthermore, some evaluations were conducted based on the relationship between web sources and their information (Yin et al. 2008). Finding duplicates in data gathered from different sources is also an important quality-assurance task in big data. Machine learning algorithms such as Gradient Boosted Decision Tree (GBDT) have been used for detecting duplicates (Wu and Song 2015). Data filtering is an approach for quality assurance through removing bad data from data sources like we did for the diffraction images. For example, Apache Samza (2016), which is a distributed stream processing framework, has been adopted for detecting and removing bad data. Nobles et al. have conducted an evaluation of the completeness and availability of electronic health record data. They identified undesirable data in datasets using machine learning algorithms such as SVM or deep learning. Since the label noise could reduce the performance of machine learning algorithms, one should improve the machine learning algorithm to handle the noise or improve the quality of the data through filtering the noise (Saez et al. 2016). Due to the massive scale of big data, automated filtering using machine learning is a preferred choice. In this article, we proposed a data filtering technique based on automated classification. Deep learning researchers are faced with the trade-off between using better deep learning architectures and better training data (Shin et al. 2016). However, building a deep learning architecture on a small training dataset is a grand challenge. Even a deep learning architecture targeted for low-shot classification requires data augmentation (Hariharan and Girshick 2016). Therefore, using a large training dataset is a more feasible approach for building a high-quality deep learning solution. However, many domain-specific applications cannot produce enough data for deep learning. Data augmentation for producing high-quality training data based on existing data is a widely adopted practice.

Some domain-specific applications can be used to produce artificial data according to the domain models, such as using aDDA for producing diffraction images of cells. Sampling from a large image is also an effective approach for producing image data (Cireşan et al. 2013; Dong et al. 2015b). In this article, we used cropping and pooling for producing a large volume of training data from an existing one. The augmented datasets were systematically evaluated in terms of veracity, fidelity, and variety. Generative models were proposed recently for producing artificial data using deep learning techniques (Dee 2017b). Although a large amount of initial data are required to produce artificial data using generative models, it is a promising technique for generating a large amount of high-quality artificial data. Through learning the transformation relation from similar datasets to produce data for low-shot learning or fine-tune an existing dataset is also a promising idea for data augmentation (Hariharan and Girshick 2016). However, poor-quality data could still be produced using these approaches. Evaluation of the produced data is still necessary to ensure the data quality.

\section{CONCLUSIONS}

Training a deep learning model normally requires a large volume of high quality training data. However, the training data may include noisy data that could impact the performance of deep learning. Therefore, it is better to remove noisy data from training data. In this article, we proposed a deep learning approach for the automated classification of data into different classes to separate noisy data from normal data. In many cases, the original training data need to be transformed to fit the input requirements of deep learning models. In other cases, new data are required through data augmentation, as the original data are insufficient in size. We discussed different data augmentation approaches and evaluated the quality of the augmented data in fidelity, variety, and veracity. 
To explain the proposed data augmentation and evaluation approaches and to demonstrate their effectiveness, we conducted a thorough experimental study on automated classification of massive diffraction images of biology cells. The proposed approaches and experience collected from this experimental study can be adopted for data augmentation and quality evaluation of datasets in other machine learning domains.

\section{REFERENCES}

2016. ADDA project. Retrieved from: https://github.com/adda-team/adda.

2016. Apache Samza. Retrieved from: http://samza.apache.org/.

2017a. Deep learning tutorial. Retrieved from: http://deeplearning.net/tutorial/lenet.html.

2017b. Open AI: Generative Models. Retrieved from: https://openai.com/blog/generative-models/.

Igor Barros Barbosa, Marco Cristani, Barbara Caputo, Aleksander Rognhaugen, and Theoharis Theoharis. 2017. Looking beyond appearances: Synthetic training data for deep CNNs in re-identification. Retrieved from: CoRR abs/1701.03153.

C. Batini, C. Cappiello, C. Francalanci, and A. Maurino. 2009. Methodologies for data quality assessment and improvement. ACM Comput. Surv. 41, 3 (2009), 16:1-16:52.

Y. Bengio. 2009. Learning deep architectures for AI. Found. Trends Machine Learn. 2, 1 (2009), 1-127.

Chris J. C. Burges. 1998. A tutorial on support vector machines for pattern recognition. Data Mining Knowl. Disc. 2 (Jan. 1998), 121-167.

L. Cai and Y. Zhu. 2015. The challenges of data quality and data quality assessment in the big data era. Data Sci. F. 14:2 (2015), 1-10.

C.-C. Chang and C.-J. Lin. 2011. LIBSVM: A library for support vector machines. ACM Trans. Intell. Syst. Technol. 2 (2011), 27:1-27:27. Issue 3.

D. C. Cireşan, A. Giusti, L. M. Gambardella, and J. Schmidhuber. 2013. Mitosis detection in breast cancer histology images with deep neural networks. In Proceedings of the International Conference on Medical Image Computing and Computerassisted Intervention. 411-418.

F. Daniel, P. Kucherbaev, C. Cappiello, B. Benatallah, and M. Allahbakhsh. 2018. Quality control in crowdsourcing: A survey of quality attributes, assessment techniques and assurance actions. Retrieved from: CoRR abs/1801.02546.

J. Ding, X. Kang, X. H. Hu, and V. Gudivada. 2017a. Building a deep learning classifier for enhancing a biomedical big data service. In Proceedings of the IEEE International Conference on Services Computing.

J. Ding, J. Wang, X. Kang, and X. Hu. 2017b. Building an SVM classifier for automated selection of big data. In Proceedings of the IEEE International Congress on Big Data.

Antonio D’Innocente, Fabio Maria Carlucci, Mirco Colosi, and Barbara Caputo. 2017. Bridging between computer and robot vision through data augmentation: A case study on object recognition. Retrieved from: CoRR abs/1705.02139.

B. Dong, L. Shao, M. Da Costa, O. Bandmann, and A. F. Frangi. 2015b. Deep learning for automatic cell detection in wide-field microscopy zebrafish images. In Proceedings of the 12th IEEE International Symposium on Biomedical Imaging (ISBI'15). $772-776$.

X. L. Dong, E. Gabrilovich, K. Murphy, V. Dang, W. Horn, C. Lugaresi, S. Sun, and W. Zhang. 2015a. Knowledge-based trust: Estimating the trustworthiness of web sources. Proc. VLDB Endow. 8, 9 (May 2015), 938-949.

J. Gao, C. Xie, and C. Tao. 2016. Big data validation and quality assuranceIssues, challenges, and needs. In Proceedings of the IEEE Symposium on Service-Oriented System Engineering (SOSE'16). 433-441.

E. Giannoulatou, S.-H. Park, D. T. Humphreys, and J. W. Ho. 2014. Verification and validation of bioinformatics software without a gold standard: A case study of BWA and Bowtie. BMC Bioinform. 15 (Suppl 16):S15 (2014).

I. Goodfellow, J. Pouget-Abadie, M. Mirza, B. Xu, D. Warde-Farley, S. Ozair, A. Courville, and Y. Bengio. 2014. Generative adversarial nets. Adv. Neural Inform. Proc. Syst. 27 (2014), 2672-2680.

V. N. Gudivada, R. Raeza-Yates, and V. V. Raghavan. 2015. Big data: Promises and problems. IEEE Comput. 48, 3 (2015), 20-23.

R. Haralick. 1971. On a texture-context feature extraction algorithm for remotely sensed imagery. In Proceedings of the IEEE Computer Society Conference on Decision and Control. 650-657.

B. Hariharan and R. B. Girshick. 2016. Low-shot visual object recognition. Retrieved from: CoRR abs/1606.02819.

M. A. Hearst, S. T. Dumais, E. Osuna, J. Platt, and B. Scholkopf. 1998. Support vector machines. IEEE Intell. Syst. Appl. 13, 4 (July 1998), 18-28.

Forrest N. Iandola, Matthew W. Moskewicz, Khalid Ashraf, Song Han, William J. Dally, and Kurt Keutzer. 2016. SqueezeNet: AlexNet-level accuracy with 50x fewer parameters and <1MB model size. Retrieved from: CoRR abs/1602.07360.

ILSVRC2012. 2012. ImageNet Large Scale Visual Recognition Challenge 2012. (2012). Retrieved from: http://www. image-net.org/challenges/LSVRC/2012/results.html. 
K. M. Jacobs, J. Q. Lu, and X. H. Hu. 2009. Development of a diffraction imaging flow cytometer. Opt. Lett. 34, 19 (2009), 2985-2987.

Jonathan Krause, Benjamin Sapp, Andrew Howard, Howard Zhou, Alexander Toshev, Tom Duerig, James Philbin, and Fei-Fei Li. 2015. The unreasonable effectiveness of noisy data for fine-grained recognition. Retrieved from: CoRR abs/1511.06789.

A. Krizhevsky, I. Sutskever, and G. E. Hinton. 2012. Imagenet classification with deep convolutional neural networks. In Proceedings of the Conference on Advances in Neural Information Processing Systems, F. Pereira, C. Burges, L. Bottou, and K. Weinberger (Eds.). 1097-1105.

K. Lee, S. A. Hasan, O. Farri, A. Choudhary, and A. Agrawal. 2017. Medical concept normalization for online user-generated texts. In Proceedings of the IEEE International Conference on Healthcare Informatics (ICHI'17). 462-469.

Nut Limsopatham and Nigel Collier. 2016. Normalising medical concepts in social media texts by learning semantic representation. In Proceedings of the 54th Meeting of the Association for Computational Linguistics. 1014-1023. DOI : https:// doi.org/10.18653/v1/P16-1096

K. S. Mehta, K. Kumar, and R. B. Jagadeeshchandra. 2014. Six-DoF UAV simulation using wind tunnel test data and its cruise mode autopilot design. In Proceedings of the International Conference on Control, Instrumentation, Communication and Computational Technologies (ICCICCT'14). 515-520.

M. Moran. 2013. Correlating the Morphological and Light Scattering Properties of Biological Cells. Ph.D. Dissertation. East Carolina University, Greenville, NC.

L. Oakden-Rayner. 2017. Exploring the ChestXray14 dataset: Problems. Retrieved from: https://lukeoakdenrayner. wordpress.com/2017/12/18/the-chestxray14-dataset-problems.

Rajat Raina, Alexis Battle, Honglak Lee, Benjamin Packer, and Andrew Y. Ng. 2007. Self-taught learning: Transfer learning from unlabeled data. In Proceedings of the 24th International Conference on Machine Learning (ICML'07). 759-766.

P. Rajpurkar, J. Irvin, K. Zhu, B. Yang, H. Mehta, T. Duan, D. Ding, A. Bagul, C. Langlotz, K. Shpanskaya, M. P. Lungren, and A. Y. Ng. 2017. CheXNet: Radiologist-level pneumonia detection on chest X-rays with deep learning. Retrieved from: CoRR abs/1711.05225 (2017).

D. Rolnick, A. Veit, S. J. Belongie, and N. Shavit. 2017. Deep learning is robust to massive label noise. CoRR abs/1705.10694.

J. A. Saez, B. Krawczyk, and M. Wozniak. 2016. On the influence of class noise in medical data classification: Treatment using noise filtering methods. Appl. Artific. Intell. 30, 6 (Jul. 2016), 590-609.

H.-C. Shin, H. R. Roth, M. Gao, L. Lu, Z. Xu, I. Nogues, J. Yao, D. Mollura, and R. M. Summers. 2016. Deep convolutional neural networks for computer-aided detection: CNN architectures, dataset characteristics and transfer learning. IEEE Trans. Medic. Imag. 35, 5 (2016), 1285-1298.

R. Sridharan. 2014. Gaussian mixture models and the EM algorithm. Retrieved from: https://pdfs.semanticscholar.org/efa8/ 185bae6608669e26b2c5ade4f00fe96becb6.pdf.

C. Sun, A. Shrivastava, S. Singh, and A. Gupta. 2017. Revisiting unreasonable effectiveness of data in deep learning era. Retrieved from: CoRR abs/1707.02968.

Christian Szegedy, Vincent Vanhoucke, Sergey Ioffe, Jonathon Shlens, and Zbigniew Wojna. 2015. Rethinking the inception architecture for computer vision. Retrieved from: CoRR abs/1512.00567.

S. K. Thati, J. Ding, D. Zhang, and X. Hu. 2015. Feature selection and analysis of diffraction images. In Proceedings of the 4th IEEE International Workshop on Information Assurance.

S. Vilkomir, J. Wang, N. L. Thai, and J. Ding. 2017. Combinatorial methods of feature selection for cell image classification. In Proceedings of the IEEE International Workshop. on Combinatorial Testing and Applications.

J. Wang. 2016. Feature Selection and Evaluation for Building an Image Classifier. (3 2016). Master's Thesis, Dept. of Computer Science, East Carolina University.

Richard Y. Wang and Diane M. Strong. 1996. Beyond accuracy: What data quality means to data consumers. F. Manage. Inf. Syst. 12, 4 (Mar. 1996), 5-33.

Peter Warden. 2018. Why you need to improve your training data, and how to do it. Retrieved from: https://petewarden. com/2018/05/28/why-you-need-to-improve-your-training-data-and-how-to-do-it/.

C. H. Wu and Y. Song. 2015. Robust and distributed web-scale near-dup document conflation in Microsoft Academic Service. In Proceedings of the IEEE International Conference on Big Data (Big Data'15). 2606-2611.

X. Yin, J. Han, and P. S. Yu. 2008. Truth discovery with multiple conflicting information providers on the web. IEEE Trans. Knowl. Data Eng. 20, 6 (June 2008), 796-808.

J. Zhang, Y. Feng, M. S. Moran, J. Q. Lu, L. V. Yang, Y. Sa, N. Zhang, L. Dong, and X. H. Hu. 2013. Analysis of cellular objects through diffraction images acquired by flow cytometry. Optics Exp. 21 (2013), 24819-24828.

M. Zhang. 2015. An Experimental Study for Automated Classification of Diffraction Images using Deep Learning. (11 2015). Master's Thesis. Dept. of Computer Science, East Carolina University, Greenville, NC.

Received July 2018; revised January 2019; accepted March 2019 